The dollar is deflated

and we must "make it" quickly

queued up outside your door

listening to you yell,

"Doucement, doucement."

We will miss you,

your friends from Montmartre, your Edith Piaf rendition,

\title{
the stories:
}

that there isn't a desert

in Africa with as many

soft rilles as yours,

the camel driver you laid

on the way back from Oran-

and the night you bet 100 francs

you could flex dice

out of your breasts to the bar

coming up seven or eleven.

We lost 1000 francs that night.

As for myself,

they broke the monotony

of your skillful cunt. 